\title{
Epidural analgesia during labour: its influences on pain relief, progress of labour, mode of delivery, maternal and foetal
}

\author{
M. Suneetha, A. Saritha* \\ Department of Obstetrics and Gynecology, Santhiram Medical College and General Hospital, Nandyal, Andhra \\ Pradesh, India \\ Received: 28 July 2019 \\ Revised: 03 December 2019 \\ Accepted: 12 December 2019 \\ *Correspondence: \\ Dr. A. Saritha, \\ E-mail: sathishkumard11@gmail.com \\ Copyright: (c) the author(s), publisher and licensee Medip Academy. This is an open-access article distributed under \\ the terms of the Creative Commons Attribution Non-Commercial License, which permits unrestricted non-commercial \\ use, distribution, and reproduction in any medium, provided the original work is properly cited.
}

\begin{abstract}
Background: Today, the availability of regional anaesthesia for labour is considered a reflection of standard obstetric care. This study is to be conducted in a tertiary care centre with all facilities available for proposed end of proving that epidural analgesia is a safe and effective method for both parturient and the foetus in abolishing pain during labour.

Methods: A total of 60 parturients were studied. they were randomly divided into two groups Group 1 includes 30 parturients. In this group parturient received epidural analgesia. The loading dose consisted of 10 ml of Bupivacaine $0.1 \%$ and Fentanyl $0.0002 \%$ (20 mcg). The top up doses were $10 \mathrm{ml}$ of $0.1 \%$ Bupivacaine and Fentanyl $0.0002 \%$, administered whenever the parturient complained of pain. When parturients enters into second stage a further 12-15 $\mathrm{ml}$ was injected with parturient in sitting position or semi-sitting position. Group 2 - (control group) Includes 30 parturients. In this group parturient was monitored without any analgesia.

Results: This prospective study was done to assess the effect of epidural analgesia on the progress of labour and its outcome, to evaluate its efficacy as an analgesic technique and to study the maternal and fetal outcome. The total number of 60 parturients were selected and randomly categorized into two groups. CASE-Those who received epidural analgesia, CONTROL-Those who did not receive any analgesia.

Conclusions: Epidural analgesia provides a versatile method of administering effective and satisfactory pain relief to parturient women. The technique should not be considered as a single entity, because the type and the dose of epidural medication can be altered as needed.
\end{abstract}

Keywords: Bupivacaine, Epidural analgesia, Fentanyl

\section{INTRODUCTION}

The American College of Obstetricians and Gynecologists (ACOG) and the American Society of Anesthesiologists (ASA) state, "There is no other circumstance where it is considered acceptable for an individual to experience untreated severe pain, amenable to safe intervention, while under a physician's care. In the absence of a medical contraindication, maternal request is a sufficient medical indication for pain relief during labor."1

Even today, misconceptions and confusions still exist among the public, physicians, nurses and amp; midwives regarding pain relief during labour. There is an ongoing debate among some obstetricians, over the use of analgesia and anesthesia for labour and delivery, especially regarding epidural neuraxial blockade. 
Whether it prolongs second stage of labour, or increase the rate of instrumental delivery and caesarean section. Although recent meta-analysis suggests, that epidural analgesia, does not improve the rate of caesarean section, others still believe that it does so.

Despite all controversy, there is no doubt that for most women, child birth is associated with severe pain often exceeding all expectations. ${ }^{4}$ Pain is not a necessary accompaniment of child birth, although pain serves the important biological function of indicating the commencement of labour to the parturient, it should be effectively relieved, once it has fulfilled this task. Persistent severe pain has harmful effects on the mother and foetus sometimes. ${ }^{2}$

Neuraxial techniques still remains the" Gold Standard" of obstetric pain management, until now, there has been no single new drug to overcome the superiority of neuraxial analgesia in obstetrics. Low dose spinal-epidural regimens provide efficacious analgesia, stable maternal haemodynamics and few tolerable side effects.

The International association for study of pain (IASP) declared 2007-2008 as the "Global year against pain in women - real pain, real women". The focus was to study both acute and chronic pain in women. Labour pain was found to be a good study model for acute pain. Increasing knowledge of physiology and pharmacotherapy of pain and development of obstetric anaesthesia as a subspeciality has improved the training in obstetric anaesthesia, leading to an overall improvement in the quality of pain relief. ${ }^{3}$

Today, the availability of regional anaesthesia for labour is considered a reflection of standard obstetric care. In our country, the awareness is still lacking and except for a few centres that run a comprehensive labour analgesia, the national awareness or acceptance of pain-relieving options for women in labour virtually does not exist.

This study is to be conducted in a tertiary care centre with all facilities available for proposed end of proving that epidural analgesia is a safe and effective method for both parturient and the foetus in abolishing pain during labour.

\section{METHODS}

The present study on epidural analgesic technique for painless labour has been carried out at a tertiary care centre, in coordination with department of anaesthesia. A total of 60 parturients were studied. They were randomly divided into two groups.

\section{Group 1 - (Study group)}

Includes 30 parturients. In this group parturient received epidural analgesia. The loading dose consisted of $10 \mathrm{ml}$ of Bupivacaine $0.1 \%$ and Fentanyl $0.0002 \%(20 \mathrm{mcg})$. The top up doses were $10 \mathrm{ml}$ of $0.1 \%$ Bupivacaine and
Fentanyl $0.0002 \%$, administered whenever the parturient complained of pain. When parturients enters into second stage a further $12-15 \mathrm{ml}$ was injected with parturient in sitting position or semi-sitting position.

\section{Group 2 - (Control group)}

Includes 30 parturients. In this group parturient was monitored without any analgesia.

\section{Methods of collection of data}

- Study design: Prospective study

- $\quad$ Study period: December 2017 - June 2018

- Sample size: 60 cases who fulfilled selection criteria.

\section{Inclusion criteria}

- Primigravida with term gestation with live singleton pregnancy with vertex presentation with spontaneous onset of labour

- Aged less than 30 years

- Patient must be in established labour

- Maternal informed consent.

\section{Exclusion criteria}

- Premature rupture of membranes

- Cephalopelvic disproportion

- Intrauterine death

- All medical diseases

- Post term pregnancy

- Foetal compromise before administration of epidural analgesia

- Previous spinal surgeries

- Spinal deformities

- Bleeding disorders

Both booked and unbooked cases admitted in labour rooms were included in the study. On admission detailed history was taken. The height and weight of the cases were recorded. Maternal pulse rate and blood pressure recorded, and general condition examined thoroughly.

Obstetric examination was done in dorsal position. Height of the uterus in weeks, presentation, position, engagement, frequency and duration of contractions noted. Fetal heart auscultated. Per vaginum examination was done with all aseptic precautions to note the following

\section{Cervical dilation, effacement and position}

- Station of the presenting part

- Membrane status

- Pelvic assessment to rule out contracted pelvis and Cephalopelvic disproportion 
Baseline investigations were done. Ultrasound examination was done for fetal well-being, liquor contentment and placental localization. If the patient satisfies the inclusion and exclusion criteria, patient's informed consent is taken for epidural analgesia.

\section{Procedure}

The procedure was explained to the patient. An $18 \mathrm{G}$ cannula started and all patient were preloaded with 500 $\mathrm{ml}$ of Ringer lactate. Patient is positioned in either sitting or lying on her side with her knees and hips flexed (Figure 1).

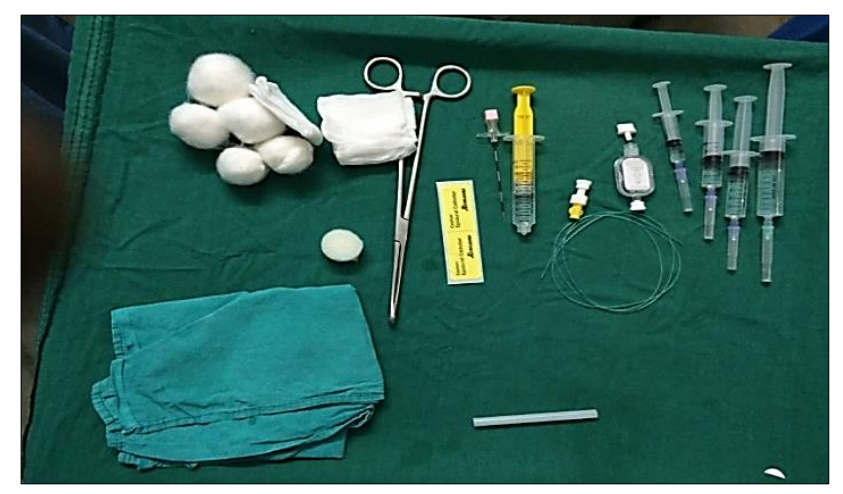

Figure 1: Requirements for epidural analgesic technique.

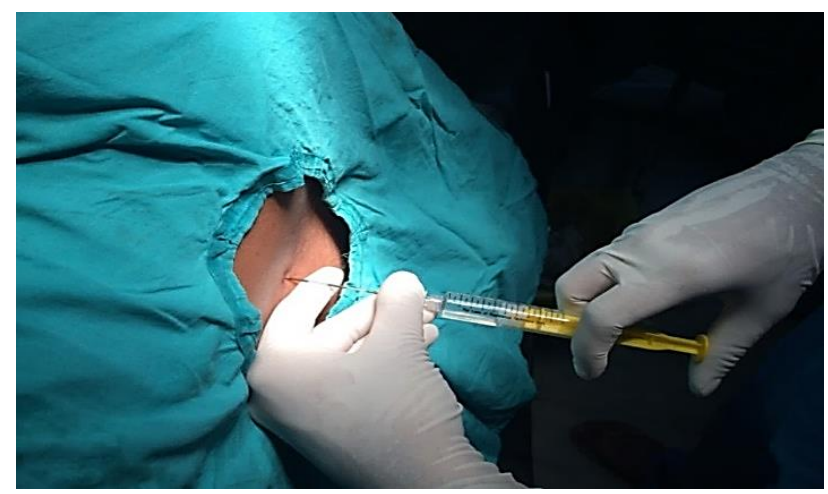

Figure 2: Epidural analgesia position.

Under aseptic precautions, multihole catheter is inserted via a specially designed16-18G Tuohy needle into epidural space at either L 2-3 or L 3-4 intervertebral space. The epidural space is located by incremental or continuous advancement of the needle using a loss of resistance syringe filled with saline or air (Figure 2). About 2-4 cm of catheter is left in the identified space and the local anaesthetic ( $10 \mathrm{ml} 0.125 \%$ bupivacaine) is administered via a filter through this catheter, as often as required for the duration of labour (Figure 3). ${ }^{4}$

The dose is given once the patient is in established labour. After the dose was given, the level of blockade was assessed by pin-prick technique and the upper level of sensory blockade was maintained at T10 level. ${ }^{5}$

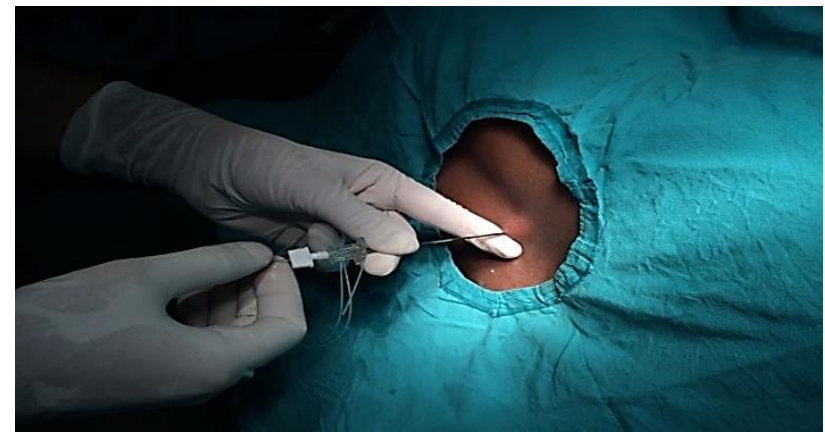

Figure 3: epidural analgesic technique.

\section{Monitoring}

\section{Maternal monitoring}

Maternal blood pressure, pulse rate were monitored every 1-2 min for first 10 minutes. Then every 5-10 minutes for subsequent 30 minutes and later every half hour.

\section{Fetal monitoring}

The fetal heart rate was assessed by doppler every $15 \mathrm{~min}$ in $1^{\text {st }}$ stage of every $5 \mathrm{~min}$ in $2^{\text {nd }}$ stage. Colour of amniotic fluid on the perineal pad was checked periodically to detect meconium stained amniotic fluid when membrane was ruptured.

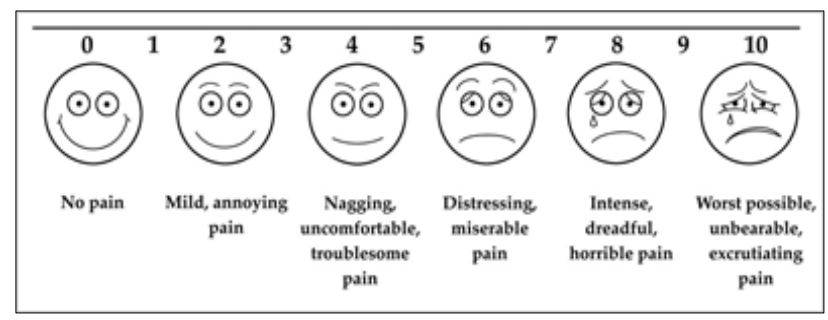

Figure 4: Visual analogue pain scale.

\section{Pain scoring}

The pain experienced by the parturient was assessed by a visual analoguepain scale (VAPS) (Figure 4).

The sedation was assessed by

\section{Wilson grading}

1. Awake, alert

2. Alert, drowsy

3. Sedation arousable by verbal commands

4. Sedation arousable by mild physical pain

5. Not arousable by pain

\section{Motor blockade}

Motor blockade assessed using the BROMAGE criteria 
$0=$ ability to move legs

$1=$ ability to move toes and partially move knees

$2=$ ability to move toes but not knees

$3=$ inability to move toes or knees

Care taken so as to ensure that bladder does not become overly distended. If parturient unable to micturate spontaneously, catheter passed and bladder drained. A partogram was maintained noting the rate of cervical dilatation, position and station of the head in relation to duration of time. Uterine contraction and fetal heart rate were noted every 15 minutes for first stage and after every contraction in second stage. Duration of each stage was recorded. Time of onset of analgesia, duration of analgesia and number of top up's required were noted. Parturients with no bearing down efforts were encouraged to bear down during contraction. Augmentation with oxytocin was used if indicated. Any maternal failure of secondary forces was assisted by outlet forceps or lower segment caesarean section was done if indicated.

The mode of delivery and general condition of the mother along with vital data were noted immediately after delivery and for one hour after delivery. Any maternal side effects were noted. After delivery the epidural catheter was removed after adding another dose for further analgesia. Catheter was checked for any damage. The puncture site was cleaned with antiseptic and covered with sterile gauze piece.

\section{Examination of the new-born}

The neonates were assessed with Apgar scores at 1 and 5 minutes and also by neurobehavioral reflexes.

\section{Statistical analysis}

The statistical analysis was performed using mean and SD deviation, calculation of percentage and student t-test was applied, to calculate P-value for any statistical significance. Where required a Chi Square test or a Fisher's exact test was performed.

\section{RESULTS}

This prospective study was done to assess the effect of epidural analgesia on the progress of labour and its outcome, to evaluate its efficacy as an analgesic technique and to study the maternal and fetal outcome. The total number of 60 parturients were selected and randomly categorized into two groups.

CASE - Those who received epidural analgesia

CONTROL - Those who did not receive any analgesia

\section{Age}

The parturients in both groups are comparable as regards to their age distribution. The p-value is insignificant (Table 1). In the cases means $21.63 \pm 1.63$, minimum value 19 , maximum 26 , in controls mean $21.63 \pm 1.69$, minimum value 19 , maximum value 26 . The P-value which is given in Table 1 is statistically insignificant.

\section{Mean duration of active phase of first stage of labour}

The P-value calculated by Unpaired t-test was 0.8071 , which statistically insignificant (Table 2). In cases group mean value in minutes $259.46 \pm 56.36$. In control group 255.54 \pm 63.27 .

Table 1: Mean age of both groups.

\begin{tabular}{|lllll|}
\hline & Mean & Minimum & Maximum & p-value \\
\hline Case & $21.63 \pm 1.63$ & 19 & 26 & 0.7149 \\
\hline Control & $21.69 \pm 1.69$ & 20 & 26 & \\
\hline
\end{tabular}

Table 2: mean duration of active phase of first stage of labour (in minutes).

\begin{tabular}{|lll|}
\hline Group & Mean (minutes) & p-value \\
\hline Cases & $259.46 \pm 56.36$ & 0.8071 \\
\hline Controls & $255.54 \pm 63.27$ & \\
\hline
\end{tabular}

\section{Duration of second stage of labour}

When calculated for significance by unpaired t-test the Pvalue was 0.0031 , which is statistically very significant (Table 3). Duration of second stage of labour in case

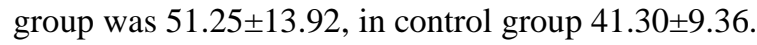

\section{Duration of third stage of labour}

The P-value calculated by unpaired t-test was 0.9040 . Thus, the difference in the duration of third stage of labour was insignificant (Table 4). Duration of third stage of labour in case group was 5.71 \pm 0.98 , in control group was $5.78 \pm 1.12$.

Table 3: Mean duration of second phase of labour.

\begin{tabular}{|lll|}
\hline Group & Mean (minutes) & p-value \\
\hline Case & $51.25 \pm 13.92$ & 0.0031 \\
\hline Control & $41.30 \pm 9.36$ & \\
\hline
\end{tabular}


Table 4: Duration of third stage of labour.

\begin{tabular}{|lll|}
\hline Group & Mean (minutes) & P-value \\
\hline Case & $5.71 \pm 0.98$ & \multirow{2}{*}{0.9040} \\
\hline Control & $5.78 \pm 1.12$ & \\
\hline
\end{tabular}

\section{Oxytocin augmentation}

Attention was paid to correct inefficient uterine action early in labour with oxytocin infusion. Benefits of routine oxytocin infusion in second stage in terms of shorter duration, short period of expulsive efforts and few instrumental deliveries are well established (Table 5).

Table 5: Oxygen augmentation.

\begin{tabular}{|ll|}
\hline & Oxytocin augmented \\
\hline Case & 27 \\
\hline Control & 26 \\
\hline
\end{tabular}

Oxytocin augmented cases in case control group was 27, in control group 26 cases of oxytocin augmented were seen. (Table 5).

Table 6: Mode of delivery in this study among both groups.

\begin{tabular}{|lll|}
\hline Mode of delivery & Case & Control \\
\hline FTNVD & $22(73.33 \%)$ & $25(83.33 \%)$ \\
\hline Instrumental delivery & $6(20 \%)$ & $3(10 \%)$ \\
\hline Em LSCS & $2(6.67 \%)$ & $2(6.67 \%)$ \\
\hline
\end{tabular}

\section{Mode of delivery}

In case group, FTNVD were $22(73.33 \%)$, instrumental delivery 6 (20\%), em LSCS were $2(6.67 \%)$ (Table 6).

Table 7: Indication of instrumental delivery in the present study.

\begin{tabular}{|lll|}
\hline & Case & Control \\
\hline Fetal distress & 2 & 2 \\
\hline Insufficient bearing down & 3 & 1 \\
\hline Direct occipito posterior position & 1 & 0 \\
\hline Prolonged $2^{\text {nd }}$ stage & 0 & 0 \\
\hline
\end{tabular}

\section{Indications for instrumental delivery}

The indications for instrumental delivery in the epidural group were two cases of fetal distress in the second stage of labour due to cord around the neck. Three were due to insufficient bearing down in the second stage and forceps were applied for maternal relief and fetal well-being. In one case there was direct occipito posterior position wherein face to pubis delivery was conducted (Table 7).

In the control group in two cases forceps were applied due to fetal distress, one for insufficient bearing down.

\section{Indication for caesarian section}

The indication for caesarean section in the study group was fetal distress for one case and secondary arrest with direct occipito-posterior position for the second case. whereas in the control group the indication for one case was fetal distress due to cord around the neck and for the other fetal distress with meconium stained liquor for the other. None of the cases had prolonged second stage of labour as shown in Table 8.

Table 8: Indication of caesarean section in the present study.

\begin{tabular}{|lll|}
\hline & Case & Control \\
\hline Fetal distress & 1 & 2 \\
\hline Secondary arrest & 1 & 0 \\
\hline Prolonged $2^{\text {nd }}$ stage & 0 & 0 \\
\hline
\end{tabular}

\section{Birth weight}

The P-value calculated by unpaired t-test was 0.3075 which is statistically insignificant (Table 9). Birth weight in case group were $2.75 \pm 0.29$. In control group, $2.68 \pm 0.28$.

Table 9: Mean birth weight in both groups.

\begin{tabular}{|lll|}
\hline Group & Mean & P-value \\
\hline Case & $2.75 \pm 0.29$ & \multirow{2}{*}{0.3075} \\
\hline Control & $2.68 \pm 0.28$ & \\
\hline
\end{tabular}

\section{Apgar at 1 minute}

The mean of apgar at one minute for epidural and control group are $7 \pm 0.91$ and $6.93 \pm 1.03$ respectively. The $\mathrm{P}$ value calculated by unpaired t-test was 0.7863 , which is statistically insignificant as shown in Table 10.

Table 10: Apgar scores at 1 min the present study.

\begin{tabular}{|llll|}
\hline Apgar & Case & Control & Total \\
\hline 4 & $1(3.33 \%)$ & $2(6.67 \%)$ & 3 \\
\hline 5 & $1(3.33 \%)$ & $0(0 \%)$ & 1 \\
\hline 6 & $3(10 \%)$ & $4(13.33 \%)$ & 7 \\
\hline 7 & $17(56.67 \%)$ & $15(50 \%)$ & 32 \\
\hline 8 & $8(26.67 \%)$ & $9(30 \%)$ & 17 \\
\hline
\end{tabular}

\section{Apgar at 5 minutes}

The mean of Apgar at five minutes for epidural and control group are $8.47 \pm 0.78$ and $8.53 \pm 0.73$ respectively. The P-value calculated by unpaired t-test was 0.7331 , which is statistically insignificant (Table 11).

\section{Maternal side effects}

In our study hypotension was seen in $2(6.67 \%)$ patients, $1(3.33 \%)$ complained of pruritis, 1 complained of 
vomiting $(3.33 \%)$ and $1(3.33 \%)$ had a $3^{\text {rd }}$ degree perineal tear with forceps assisted delivery.

Table 11: Apgar scores at $5 \mathrm{~min}$ in the present study.

\begin{tabular}{|llll|}
\hline Apgar & Case & Control & Total \\
\hline 7 & $4(13.33 \%)$ & $3(10 \%)$ & 5 \\
\hline 8 & $9(30 \%)$ & $9(30 \%)$ & 17 \\
\hline 9 & $16(53.33 \%)$ & $17(56.67 \%)$ & 35 \\
\hline 10 & $1(3.33 \%)$ & $1(3.33 \%)$ & 3 \\
\hline
\end{tabular}

\section{Motor block}

Motor blockade following epidural analgesia was assessed using bromage scale. None of the parturients had motor blockade.

\section{DISCUSSION}

The ideal labour analgesic technique should be effective, safe for mother and foetus, should be easy to administer, should provide consistent, predictable and rapid onset of analgesia in all stages of labour, should be devoid of motor blockade and should preserve the stimulus for expulsive efforts during the second stage of labour. ${ }^{5}$

Labour pain is a subjective experience with sensory and emotional components. Thus, the perception of pain and response to it, varies from one parturient to the other. ${ }^{6}$

Lumbar epidural technique as a means of obstetric pain relief has established its supremacy. ${ }^{5}$ Epidural analgesia is used principally for pain relief during labour. It is estimated that some $20 \%$ of all the parturients now receive epidural analgesia for pain relief in labour. ${ }^{7}$ Safe and effective relief of pain during labour and delivery accomplished by the skillful use of epidural analgesia prevents the stress response in the mother. Maternal hypoxemia, hypocapnia, catecholamine secretion leading to uterine hypoperfusion, foetal hypoxia and acidosis are avoided. ${ }^{8}$

Obstetricians and anesthesiologists have always feared that incidence of instrumental deliveries in women receiving epidural analgesia could be higher than in those who do not receive it. ${ }^{6}$ Studies have revealed that the threshold of the obstetricians to perform assisted delivery is definitely lower when epidural analgesia is already present. $^{9}$

Thus, the present study was undertaken to evaluate the effect of epidural analgesia on labour and its outcome.

\section{Analgesic drugs used}

Bupivacaine still remains the most often used local anaesthetic. Various workers have used varying concentrations of bupivacaine. Undiluted bupivacaine $(0.5 \%)$ was popular for initiation and maintainance of labour analgesia. ${ }^{10}$ However it caused dense motor blockade and interference with maternal awareness of contractions. ${ }^{11}$ Despite providing excellent pain relief in labour, epidural analgesia using local anaesthetics alone produces motor block in $85 \%$ of the patients and associated with a prolonged second stage and an increased incidence of instrumental delivery. ${ }^{12}$

In an attempt to reduce this undesirable effect, efforts were made to reduce the concentration and total dose of local anaesthetics used. Adjuvants like fentanyl were added so as to decrease the bupivacaine concentration to as low as $0.0625 \%$. Bupivacaine in the concentration of $0.125 \%$ were used by Bleyert, Kahn et al, Guisasola. They observed there was significant avoidance of motor blockade.

There was no prolongation of second stage of labour and no difference in the mode of delivery. Li et al, studied the efficacy of bupivacaine by reducing the concentration from $0.25 \%$ up to $0.0625 \%$ as bolus.

Purdy et al compared $0.5 \%, 0.375 \%, 0.25 \%$ bupivacaine given as bolus. They observed that by reducing the concentration, quality of analgesia did not differ. However lower concentration of local anaesthetics minimised or prevented the motor block.

However, the use of low concentrations of bupivacaine provides suboptimal, short lived analgesia when used alone. Epidural opiods offer the possibility of analgesia without motor block, but when used alone do not provide satisfactory analgesia throughout labour. Addition of an opioid to local anaesthetic can provide effective analgesia with bupivacaine sparing and reduction in motor block. In this study bupivacaine in the concentration of $0.125 \%$ was used.

\section{CONCLUSION}

Epidural analgesia provides a versatile method of administering effective and satisfactory pain relief to parturient women. The technique should not be considered as a single entity, because the type and the dose of epidural medication can be altered as needed. Although child birth has become much safer for mother and neonate, there is still chance for improvement. Existing evidence suggests that women should not avoid epidural analgesia for fear of neonatal harm or caesarean delivery. There is statistically significant increased duration of the second stage of labour. There is no increase in the rate of caesarean section. Maximum numbers of deliveries were spontaneous in this study. There was no significant difference in the rate of instrumental vaginal delivery. Apgar scores were good, implying no adverse effects on neonatal outcome. It is possible that adjustments in epidural and obstetric care may minimize any untoward effects, requiring a team approach to the management of the $2^{\text {nd }}$ stage. Current interest in obstetric analgesia is focusing on refined 
epidural techniques or on the use of alternative local anaesthetic/opiate mixtures. The evidence should be made available to women considering pain relief in labour. The decision about whether to have an epidural should then be made in consultation between the woman and her career.

Funding: No funding sources Conflict of interest: None declared

Ethical approval: The study was approved by the Institutional Ethics Committee

\section{REFERENCES}

1. Pain relief during labour: ACOG Committee Opinion no.295: American College of Obstetricians and Gynaecologists. Obstet Gynecol. 2004;104:213.

2. Halpern SH, Leighton BL, Ohlsson A, Barrett JF, Rice A. Effect of epidural vsparenteral opioid analgesia on the progress of labor. A meta-analysis. JAMA. 1998;280:2105-10.

3. Thorp JA, Hu DH, Albin RM, McNitt J, Meyer BA, Cohen GR, et al. The effect of intrapartum epidural analgesia on nulliparous labor. A randomized, controlled, prospective trial. AM J Obstet Gynecol. 1993;169:851-8.

4. Stolte K. A comparison of women's expectation of labor with the actual event. Birth. 1987;14:99-103.

5. Bonica JJ. The nature of pain in parturition: Van Zundert A, Ostheimer GW, editors. Pain relief and
Anesthesia in Obstetrics. New York: Churchill Livingstone; 1996:1952.

6. Hawkins JL. Epidural analgesia for labour and delivery. N Engl J Med. 2010;362:1503-10.

7. Wong CA. Advances in labor analgesia. Int $\mathbf{J}$ Womens Health. 2009;1:139-54.

8. Cohen J. Doctor James Young Simpson, Rabbi Abraham De Sola, and Genesis, 1996. Chapter 3, verse 16. Obstet Gynecol. 1996;88:895-8.

9. Snow J. On administration of chloroform in during parturition. Assoc Med J. 1853;1:500-2.

10. Lieberman E, O'Donohue C. Unintended effects of epidural analgesia during labor: A systematic review. Am J Obstec Gynecol. 2002;186:S31-68.

11. Erving HW. "The Discoverer of Anæsthesia: Dr. Horace Wells of Hartford". Yale J Biol Med. 1933;5(5):421-30.

12. Minnitt RJ. Self-administration analgesia for the midwifery of general practice. $\mathrm{Br} \mathrm{J}$ Anesth. 1934; $11: 148-52$.

Cite this article as: Suneetha M, Saritha A. Epidural analgesia during labour: its influences on pain relief, progress of labour, mode of delivery, maternal and foetal. Int J Reprod Contracept Obstet Gynecol 2020;9:555-61. 